\title{
Iron Deficiency as Cause of Dysphagia and Burning Mouth (Plummer-Vinson or Kelly-Patterson Syndrome): a Case Report
}

\author{
Vladimíra Radochová1,*, Radovan Slezák ${ }^{1}$, Jakub Radocha²
}

\section{ABSTRACT}

The clinical presentation of iron deficiency can be very heterogeneous, including various oral and other mucosal problems. Here, in this case, we report the patient with burning mouth and dysphagia symptoms where iron deficiency was found to be the underlying cause after several months of investigations. This clinical syndrome is called Plummer-Vinson syndrome. It is sporadic with an incidence less than $0.1 \%$ of patients suffering from iron deficiency anemia.

\section{KEYWORDS}

iron deficiency; anemia; dysphagia; burning mouth

\section{AUTHOR AFFILIATIONS}

${ }^{1}$ Department of Dentistry, Faculty of Medicine in Hradec Králové, Charles University, and University Hospital Hradec Králové, Hradec Králové, Czech Republic

2 4th Department of Internal Medicine-Hematology, Faculty of Medicine in Hradec Králové, Charles University, and University Hospital Hradec Králové, Hradec Králové, Czech Republic

* Corresponding author: Department of Dentistry, University Hospital, Sokolská 581, 50005 Hradec Králové,Czech Republic; e-mail: vladimira.radochova@fnhk.cz 


\section{BACKGROUND}

Iron deficiency anemia (IDA) is one of the most common anemias worldwide (IDA) and is frequently accompanied by other symptoms not typically associated with anemic syndrome (1). As a part of the clinical picture, patients with IDA may present with burning mouth syndrome, chronic glossitis, pale mucosal membranes etc. Various other symptoms include hair loss, nail changes, pica and rarely dysphagia (1). Dysphagia associated with IDA was described by Donald Ross Patterson and Adam Brown Kelly, both British laryngologists, in 1919. This clinical syndrome of IDA and dysphagia called Plummer-Vinson syndrome (PVS) is, however named after Henry Stanley Plummer and Porter Paisley Vinson, both physicians of Mayo Clinic, USA (2). PVS is a rare condition and consists of glossitis, atrophic oral mucosa, dysphagia and anemia. Strictures of the upper esophagus are frequently described as a part of PVS. The majority of reported cases were women (up to $89 \%$ ) around 5 th decennium (3). Here we present a case of a female patient presenting with dysphagia and burning mouth with long time unknown cause of both symptoms.

\section{CASE PRESENTATION}

A 65-year-old woman was referred for burning mouth to the examination to the Department of Dentistry, University Hospital Hradec Králové, Czech Republic. Her history of current complaints included one year of difficulty in swallowing of solid food, dry mouth, burning tongue especially on the tip of the tongue and angular cheilitis. She also reported chronic fatigue and shortness of breath on exertion and loss of weight of $3 \mathrm{~kg}$ per last year. No other symptoms were reported by the patient.

Her personal history includes arterial hypertension, hypercholesterolemia, gastroesophageal reflux caused by hiatal hernia and chronic pain caused by previous surgery of right femur 20 years ago. Her corresponding chronic medication included meloxicam, pregabalin, esomeprazole, atorvastatin, hydrochlorothiazide and metoprolol. She reported herself as nonsmoker without any allergies. She was retired at the time of examination and previously employed as an administrative office worker.

During previous investigations, she was seen by a number of specialists. She was repeatedly examined by otorhinolaryngologist with normal findings on clinical/ physical examination six months before diagnosis. She was scheduled for barium contrast swallow test with the conclusion of hiatus hernia and corresponding inflammatory changes. She also underwent examination by her internal medicine specialist, and ultrasound of the abdomen was performed with no significant findings, and esophageal manometry was performed with normal results. She was scheduled for esophageal $\mathrm{pH}$ measurements. Until the dental examination, no laboratory examination was done.

Extraoral clinical examination revealed bilateral angular cheilitis with central rhagades with only skin and transition zone involved (Figure 1). Other skin findings on the skull and face were normal. Lymph nodes were not enlarged. Intraoral examination showed atrophic glossitis

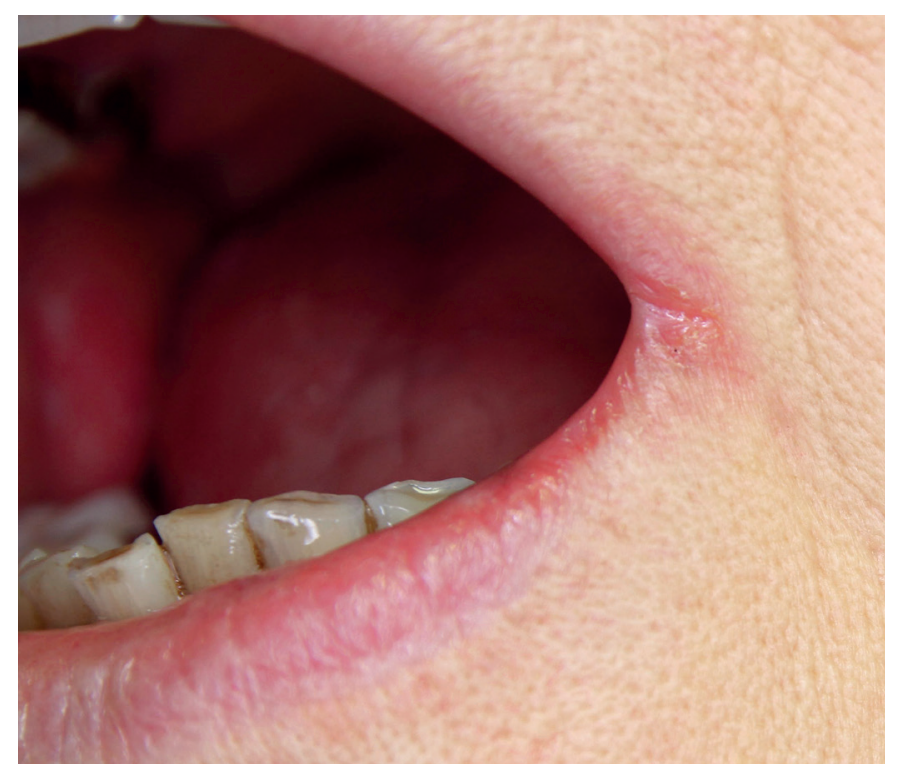

Fig. 1 Angular cheilitis.

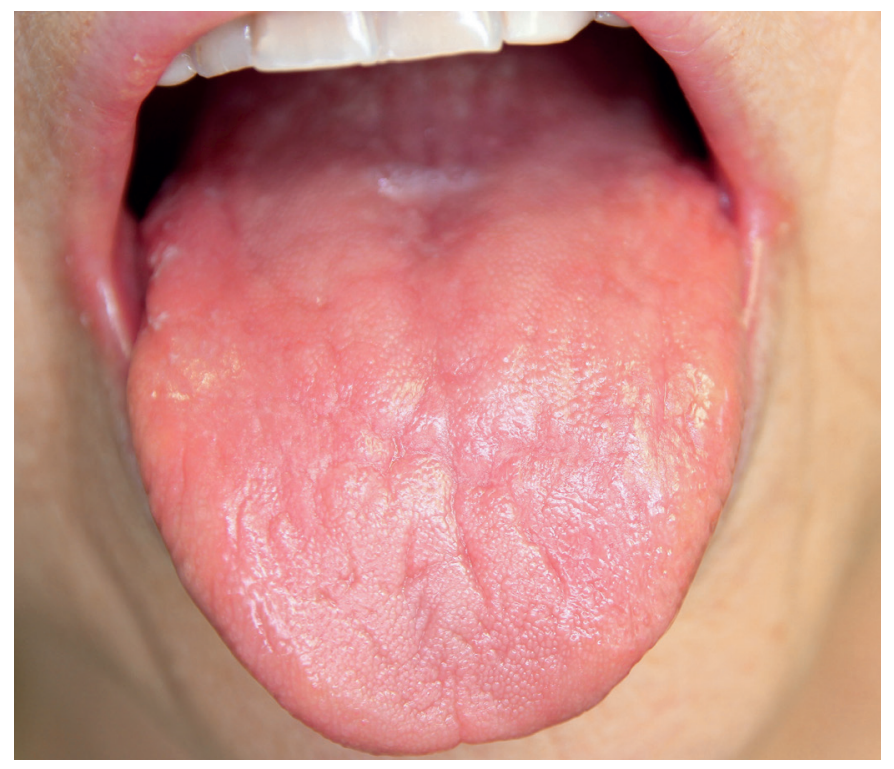

Fig. 2 Atrophic tongue mucosa.

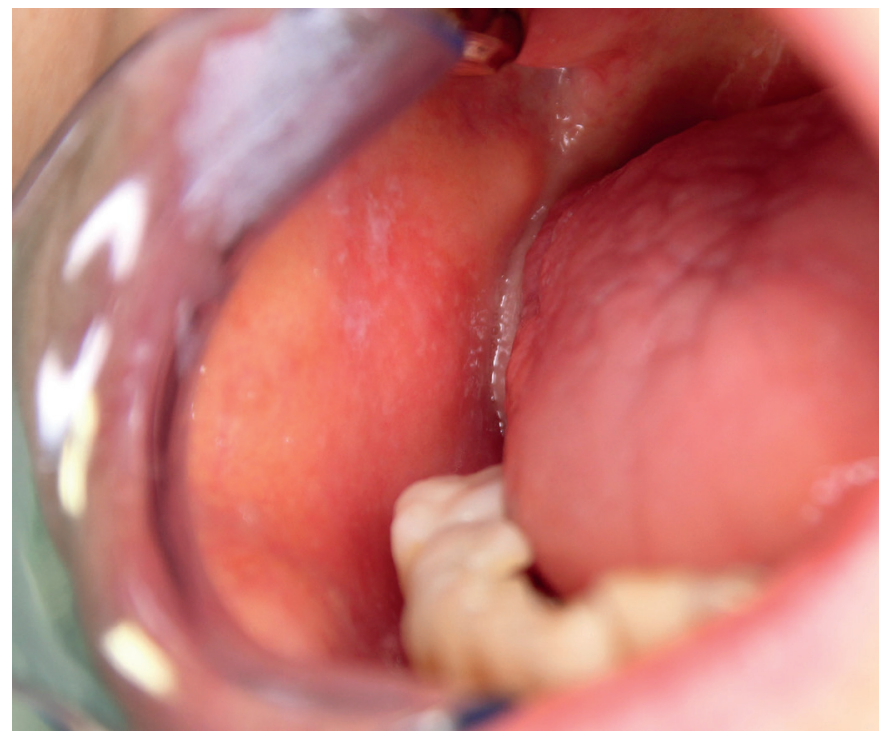

Fig. 3 Buccal erythema with candida overgrowth. 
Tab. 1 Blood count values.

\begin{tabular}{|c|c|c|c|c|}
\hline & At diagnosis & At +6 months & Unit & Normal range \\
\hline Leukocytes & 7.92 & 6.39 & $\times 10^{9} / l$ & $4.00-10.00$ \\
\hline Erythrocytes & 4.46 & 5.4 & $\times 10^{12} / /$ & $3.80-5.20$ \\
\hline Hemoglobin & 76 & 140 & $g / l$ & $120-160$ \\
\hline Hematocrit & 27.0 & 44.5 & $\%$ & $35-47$ \\
\hline Mean cell volume & 60.5 & 82.4 & $f l$ & $82.0-98.0$ \\
\hline Mean cell hemoglobin & 17.0 & 25.9 & pg & $28-34$ \\
\hline Mean cell $\mathrm{Hb}$ concentration & 0.28 & 0.32 & $\mathrm{~kg} / \mathrm{l}$ & $0.32-0.36$ \\
\hline Thrombocytes & 439 & 368 & $\times 10^{9} / \mathrm{l}$ & $150-400$ \\
\hline Ferritin & 7.0 & 38.8 & ug/l & $30-400$ \\
\hline
\end{tabular}

with loss of papillae (Figure 2). Discrete erythema with candida overgrowth (white patches) was present of both buccal mucosal surfaces (Figure 3). Other mucosal and tooth findings were unremarkable.

Complementary laboratory examinations were recommended based on history and clinical findings. Sum of resting (unstimulated) salivary flow and stimulated salivary flow (Škach's quantitative analysis of the saliva) revealed salivation of $8 \mathrm{ml} / 2 \times 15$ minutes which is at lower normal margin. Full blood count with differential blood count; ferritin, serum iron, total iron-binding capacity, vitamin B12 and folic acid levels were measured. Tongue swab was sent for mycological cultivation. Significant results are shown in Table 1. Diagnosis of IDA was established. Topical treatment for angular cheilitis with hydrocortisone, natamycin and neomycin was recommended, and the patient was referred for hematology examination. Subsequent gastroscopy showed chronic gastric inflammation without atrophy on histology and previously known hiatus hernia. Colonoscopy was performed with normal findings. No blood losses were identified. Treatment with oral iron supplementation was initiated at a dose of $80 \mathrm{mg}$ of elemental iron daily. The blood count normalized eight weeks after treatment, and iron supplies recovered six months

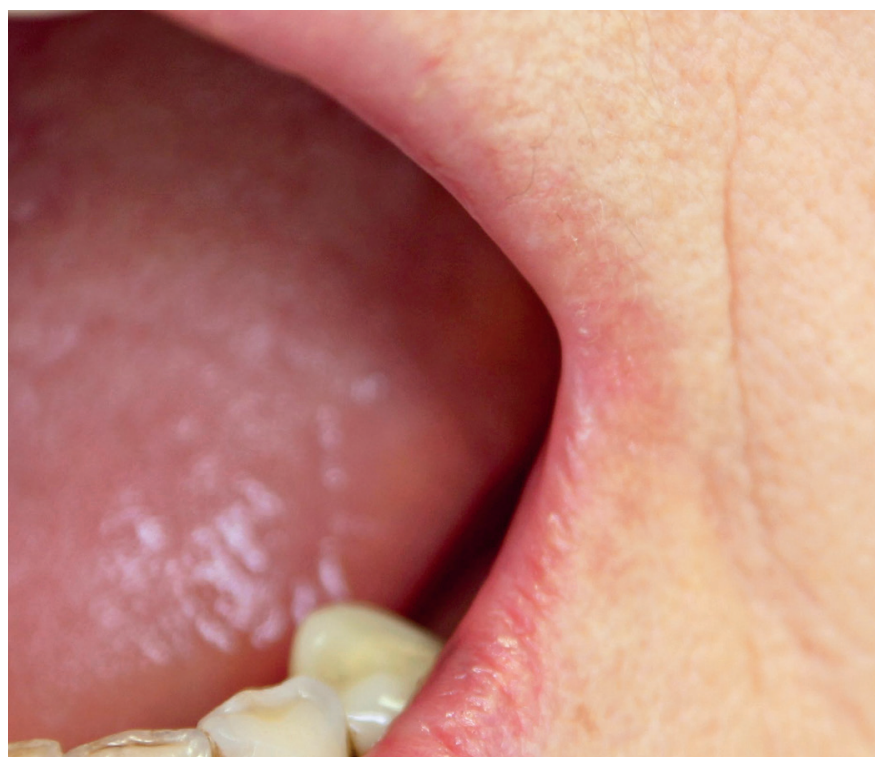

Fig. 4 Angular cheilitis after treatment. after treatment (Table 1). Patient reported improving the symptoms eight weeks after treatment initiation and did not report any symptoms after six months (Figures 4-6).

\section{DISCUSSION}

Nutritional deficiencies, including iron, are also common and still represent a significant medical challenge even in the contemporary European population. Diet habits and socioeconomic status may contribute to the development of such problems (4). Potentially malignant lesions of the gut should be considered behind each iron deficiency cause (5). Mainly central European populations show extremely unfavourable incidence and prevalence pattern of colon cancer (6).

Burning mouth syndrome is one of the most frequent signs that bring patients to dental medicine clinics. It represents a wide variety of diseases ranging from benign to severe disorders. The exact etiology of burning mouth cannot be frequently identified, but the cause may be identified in many patients (7). Nutrition deficiencies and anemia represent the frequent and often reversible cause of burning mouth. Lin et al. described a decrease

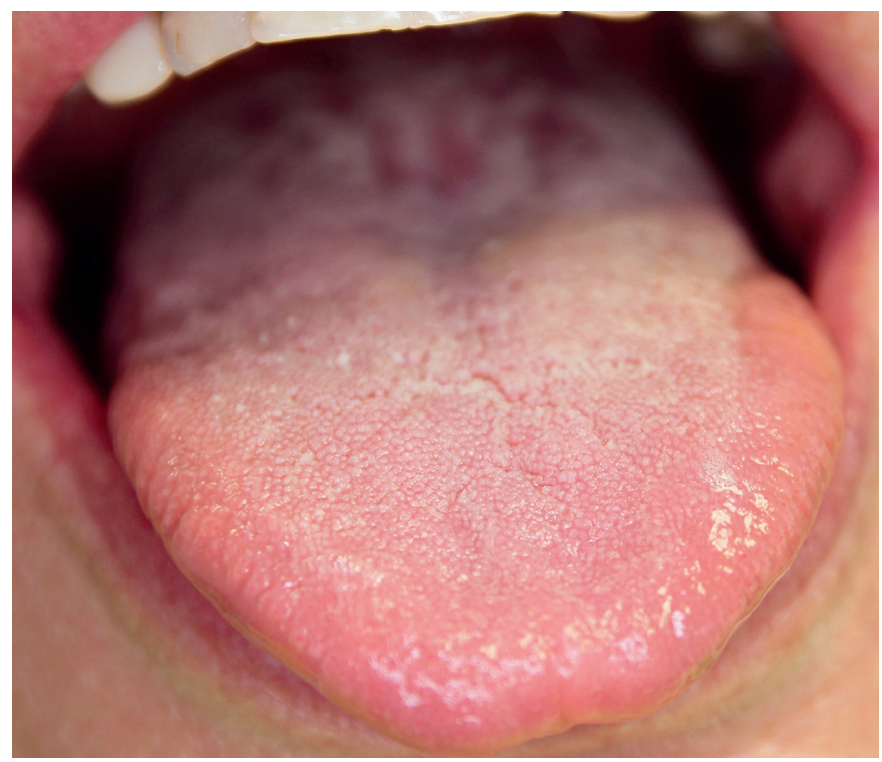

Fig. 5 Healthy tongue after treatment. 
of hemoglobin in a cohort of 399 Asian patients with the burning mouth in $22.3 \%$. $20.3 \%$ of these patients had iron deficiency, $2.5 \%$ vitamin B12 deficiency and $1.5 \%$ folic acid deficiency (8). According to Sun et al., patients with papillar atrophy more frequently have iron deficiency (26.7\%) than vitamin B12 deficiency (7.4\%) (9). Recently published data by Lu showed the prevalence of oral pseudomembranous and erythematous candidiasis around $40 \%$ of individuals in patients with iron deficiency (10). Atrophic glossitis may have a very similar clinical picture and could mask underlying deficiency (11). Reversal of symptoms by supplementation of missing nutrients has been shown to be effective in a cohort of 399 patients (5). The burning mouth disappeared within 5-10 months after supplementation of missing nutrient in virtually all affected patients, similar to our patient (12).

On the other hand, burning mouth with dysphagia is a sporadic clinical condition, and PVS is present in less than $0.1 \%$ of patients suffering from IDA (3). Typically, PVS is characterized by IDA combined with glossitis, atrophic mucosa, dysphagia and strictures of the upper esophagus (3). Pathogenesis of PVS remains unknown and how IDA contributes to the development of dysphagia has not been fully understood. Possible high turnover of the epithelial cells in upper esophagus dependent on iron storage remains a reasonable explanation (13). It has been proposed that genetic, immunologic and infectious factors may play a role (14). Strictures are based on the presence of esophageal webs that have not been seen in our patient. Our patient did not show any abnormalities in esophageal motility as well. Not all patients with symptoms compatible with PVS need to develop webs, and vice versa, not all patients with esophageal webs suffer from dysphagia and fulfil criteria for PVS $(15,16)$. Symptoms completely disappeared in our patient after treatment which does not always happen in all patients with PVS. The most frequents reasons for IDA in patients with PVS are menstrual blood loss, chronic gastrointestinal bleeding or hiatal hernia (as in our case). By far the most frequently, the association with celiac disease has been repeatedly reported (3). This fact

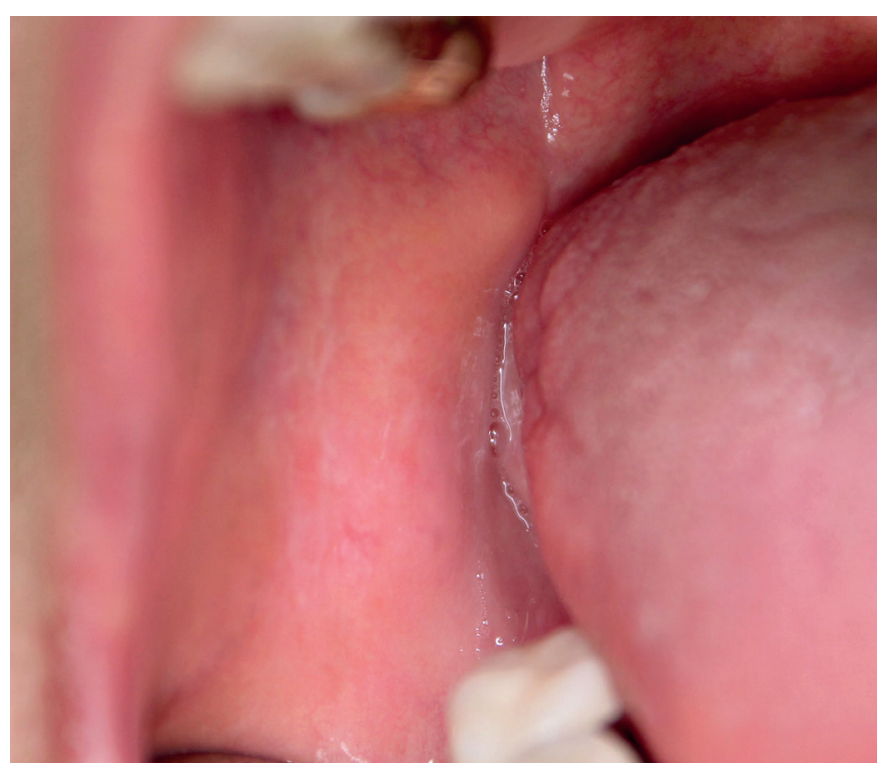

Fig. 6 Healthy buccal mucosa after treatment may be explained either by the presence of refractory iron deficiency in some patients with the celiac disease caused by impaired nutrient absorption or by the contribution of autoimmunity to development of PVS. Other autoimmune disorders were also reported in patients with PVS such as autoimmune thyroiditis and rheumatoid arthritis. Burning mouth and dysphagia could also be a sign of underlying Sjögren syndrome, and diagnostic procedures should include sialometry as well as organ antibody testing. Prognosis of PVS is generally good with appropriate management with iron supplementation in the majority of patients. There have been reports about patients with PVS and strictures and stenosis of the upper esophagus who developed squamous cell carcinoma of the esophagus and presence of the webs probably represents a risk for developing pharyngeal cancer (17). Patients with PVS and esophageal webs and strictures should be therefore monitored for possible signs of carcinoma. Surveillance with repeated endoscopies has been suggested, but the preventive endoscopic evaluation is not confirmed (17).

\section{LEARNING OBJECTIVES}

Both dysphagia and burning mouth should bring attention to possible iron deficiency and other nutritional deficiencies. Dysphagia and burning mouth resolve after iron supplementation in the majority of patients.

\section{ACKNOWLEDGEMENTS}

The work was supported by the project PROGRES Q40/08 and Q40/13 (Charles University, Faculty of Medicine in Hradec Králové) and by the Czech Health Research Council, Ministry of Health of the Czech Republic and MH CZ DRO (UHHK, 00179906).

\section{CONFLICTS OF INTEREST}

None declared.

\section{REFERENCES}

1. Lopez A, Cacoub P, Macdougall IC, Peyrin-Biroulet L. Iron deficiency anaemia. Lancet 2016; 387: 907-16.

2. Plummer HS. Diffuse dilatation of the esophagus without anatomic stenosis (cardiospasm): a report of ninety-one cases. J Am Med Assoc 1912; 26: 2013-5.

3. Novacek G. Plummer-Vinson syndrome. Orphanet J Rare Dis 2006; 1 : 36.

4. Huffman FG, Vaccaro JA, Zarini GG, Biller D, Dixon Z. Inadequacy of micronutrients, fat, and fiber consumption in the diets of Haitian-, African- and Cuban-Americans with and without type 2 diabetes. Int J Vitam Nutr Res 2012; 82: 275-87.

5. Shine JW. Microcytic anemia. Am Fam Physician 1997; 55: 2455-62.

6. Zavoral M. Colorectal cancer screening in the Czech Republic. Z Für Gastroenterol. 2008; 46(Suppl 1): S29-30.

7. Sun A, Wu K-M, Wang Y-P, Lin H-P, Chen H-M, Chiang C-P. Burning mouth syndrome: a review and update. J Oral Pathol Med 2013; 42: 649-55.

8. Lin H-P, Wang Y-P, Chen H-M, Kuo Y-S, Lang M-J, Sun A. Significant association of hematinic deficiencies and high blood homocysteine levels with burning mouth syndrome. J Formos Med Assoc 2013; 112: 319-25. 
9. Sun A, Lin H-P, Wang Y-P, Chiang C-P. Significant association of deficiency of hemoglobin, iron and vitamin B12, high homocysteine level, and gastric parietal cell antibody positivity with atrophic glossitis. J Oral Pathol Med 2012; 41: 500-4

10. Lu S-Y. Perception of iron deficiency from oral mucosa alterations that show a high prevalence of Candida infection. J Formos Med Assoc 2016; 115: 619-27.

11. Terai H, Shimahara M. Atrophic tongue associated with Candida. J Oral Pathol Med. 2005; 34: 397-400.

12. Sun A, Lin H-P, Wang Y-P, Chen H-M, Cheng S-J, Chiang C-P. Significant reduction of serum homocysteine level and oral symptoms after different vitamin-supplement treatments in patients with burning mouth syndrome. J Oral Pathol Med 2013; 42: 474-9.
13. Okamura H, Tsutsumi S, Inaki S, Mori T. Esophageal web in Plummer-Vinson syndrome. The Laryngoscope 1988; 98: 994-8.

14. Tahara T, Shibata T, Okubo M, et al. A case of plummer-vinson syndrome showing rapid improvement of dysphagia and esophageal web after two weeks of iron therapy. Case Rep Gastroenterol 2014; 8: 211-5.

15. Gude D, Bansal D, Malu A. Revisiting Plummer Vinson Syndrome. Ann Med Health Sci Res 2013; 3: 119-21.

16. Nosher JL, Campbel WL, Seaman WB. The clinical significance of cervical esophageal and hypopharyngeal webs. Radiology 975; 117: 45-7.

17. Hoffman RM, Jaffe PE. Plummer-Vinson syndrome. A case report and literature review. Arch Intern Med 1995; 155: 2008-11. 